\title{
Hippocampal CA1 kindling but not long-term potentiation disrupts spatial memory performance
}

\author{
L. Stan Leung ${ }^{1}$ and Bixia Shen
}

\author{
Departments of Physiology and Pharmacology and Clinical Neurological Sciences, The University of Western Ontario, \\ London, Canada N6A 5C1
}

\begin{abstract}
Long-term synaptic enhancement in the hippocampus has been suggested to cause deficits in spatial performance. Synaptic enhancement has been reported after hippocampal kindling that induced repeated electrographic seizures or afterdischarges (ADs) and after long-term potentiation (LTP) defined as synaptic enhancement without ADs. We studied whether repeated stimulations that gave LTP or ADs resulted in spatial performance deficits on the radial arm maze (RAM) and investigated the minimal number of ADs required for such deficits. Three experimental groups were run as follows: (1) 5 hippocampal ADs in $1 \mathrm{~d}$ (5-AD group), (2) 10 hippocampal ADs in $2 \mathrm{~d}$ (10-AD group), and (3) $12 \theta$-frequency primed-burst stimulations (PBSs) in $2 \mathrm{~d}$ in order to induce LTP without ADs (LTP group). Each experimental group was run together with a control group during the same time period. Rats were first trained in a spatial task on a radial arm maze with four of the eight arms baited, then given control or experimental treatment, and maze performance was tested in the first week (1-4 d) and fourth week (22-25 d) after treatment. Basal dendritic population excitatory postsynaptic potentials (pEPSPs) and medial perforant path (MPP)-evoked dentate gyrus population spike and polysynaptic CAl excitation were recorded before and after experimental and control treatment. Spatial memory errors, in particular reference memory errors, were significantly higher in the 10-AD kindled group than any other group on the first and fourth week after treatment. Spatial memory errors were not significantly different in the 5-AD and LTP groups as compared with any control groups at any time. Basal dendritic pEPSP in CAl was enhanced for about 1 wk after 12 PBSs, 10 ADs, or 5 ADs, while the dentate gyrus population spike and CAl polysynaptic excitation evoked by MPP was increased for up to 4 wk after 10 ADs, but not 12 PBSs. Thus, distributed alteration of multiple synaptic transmission in the entorhinal-hippocampal circuit, but not LTP at the basal dendritic synapses in CAl, may disrupt spatial performance after 10 hippocampal ADs.
\end{abstract}

Temporal lobe epileptic patients commonly complain about memory problems that are corroborated by neuropsychological testing (Thompson 1991). The memory problem may result from existing pathology (Bruton 1988) or functional changes after seizures. An episode of multiple recurrent seizures in the temporal lobe may disrupt verbal and nonverbal memory, with preservation of general intelligence for at least one day (Halgren et al. 1991).

We and others have studied the spatial memory and performance after repeated seizures in the hippocampus using the kindling model (Lopes da Silva et al. 1986; Hannesson and Corcoran 2000; Leung et al. 2000; Hannesson et al. 2001). Kindling refers to the delivery of spaced, repeated (electrical or chemical) stimulations to the brain that result in a progression of clinical signs of epilepsy, culminating in motor and spontaneous seizures (Goddard et al. 1969). Partial kindling refers to the repeated delivery of afterdischarges (ADs) or electrical seizures without motor convulsions. Kindling of the hippocampus induced spatial memory deficits, as tested on the radial arm maze (RAM) (Lopes da Silva et al. 1986; Leung et al. 1990, 1993, 1996; Leung and Shen 1991; Robinson et al. 1993; Feasey-Truger et al. 1993; Sutula et al. 1995) or water maze (Gilbert et al. 1996; Sutherland et al. 1997; Hannesson and Corcoran 2000; Hannesson et al. 2001).

If spatial learning involves changes in synaptic transmission in the hippocampus, then alteration in synaptic weights may disrupt spatial learning. Induction of long-term potentiation

\footnotetext{
${ }^{1}$ Corresponding author.

E-mail sleung@uwo.ca; fax (519) 661-3827.

Article published online ahead of print. Article and publication date are at http://www.learnmem.org/cgi/doi/10.1101/lm.66106.
}

(LTP) has yielded diverse results. Several studies showed that LTP of the perforant path to dentate gyrus (DG) synapses disrupted spatial learning (Castro et al. 1989; Barnes et al. 1994; Moser et al. 1998) but others did not (Robinson 1992; Cain et al. 1993; McNamara et al. 1993). Lack of a distributed disruption of the hippocampal circuit or lack of LTP saturation (Moser et al. 1998) may account for the difference. The alternate approach to block LTP has also yielded somewhat mixed results. An NMDA receptor antagonist, shown to block LTP in the DG, may disrupt watermaze spatial learning only if the rats had no prior maze pretraining (Bannerman et al. 1995; Saucier and Cain 1995). Further, mice lacking functional glycoprotein Thy-1 showed intact watermaze spatial learning despite a suppressed DG-LTP (NostenBertrand et al. 1996).

A metabotropic glutamate receptor agonist was reported to suppress LTP in CA1 in vivo, but did not impair a spatial watermaze task (Holscher et al. 1997). On the other hand, mice with NMDAR1 subunit knock-out selective to the CA1 area had lessspecific place cells (Tsien et al. 1996), perhaps because NMDAdependent LTP is responsible for setting the spatial fields of place cells (Muller et al. 1996; Dragoi et al. 2003). In contrast to the DG, we are not aware of any report of spatial learning after LTP induction in CA1. Thus, one of our goals was to study the spatial performance of rats after LTP in CA1. We selected the basal dendritic afferent to CA1 pyramidal cells that is known to express robust LTP that lasts several days (Leung et al. 1992, 2003; Leung and Shen 1995).

In this study, we were primarily interested in the longlasting effects of seizures that last for days and weeks. In rats that were trained on an 8-arm RAM, retention deficits were found for a duration of $4 \mathrm{wk}$ after 15 repeated hippocampal ADs (Leung et 
al. 1994). In another study, 10 ADs were sufficient to disrupt performance on a place but not a cue task on the RAM (Leung et al. 1996). In the present study, our first objective was to investigate whether 5 hippocampal ADs, as compared with 10 ADs, induced long-lasting spatial RAM deficits. Our second objective was to study whether changes in hippocampal CA1 synaptic transmission, induced by LTP without ADs, was correlated with spatial deficits on the RAM.

\section{Materials and Methods}

\section{Experiment 1. Partial hippocampal kindling with 10 ADs and spatial performance}

Sixteen male Long Evans hooded rats, initially of 160-180 g were used. The rats were first trained on the RAM. During RAM trials, the rats were maintained at $\sim 85 \%$ of their ad libitum weight, adjusted for rats of the same age. The physical characteristics of the 8-arm RAM have been described (Leung et al. 1990). Various extramaze cues including bookshelves, wall posters, a ribbon, and a door could be seen from inside the maze. For each rat, four randomly selected arms were selected and baited with food; no more than two adjacent arms were baited. The partially baited RAM was preferred over the all-arms-baited RAM to prevent stereotypic behaviors (e.g., visiting arms sequentially; see Lanke et al. 1993) and to allow operational measurements of reference and working memory (Olton et al. 1979). In the first six sessions, food pellets were placed along the arms as well as at the end of the arms; performance during these trials were not scored. Starting on the seventh session, two Noyes food pellets ( $45 \mathrm{mg}$ each) were placed in the sunken food wells at the end of the arms, and a rat was permitted to remain on the maze until all four baited arms were entered, 16 choices were made, or 5 min had elapsed. A reference memory (RM) error was defined as the selection of an unbaited arm and a working memory (WM) error was defined as the revisit of any previously entered arm. A choice score was defined as the number of correct (baited) arms entered in the first four choices. The time of completing the maze was also measured. Two trials were run per day, with the trials separated by at least $3 \mathrm{~h}, 5 \mathrm{~d} / \mathrm{wk}$, until the performance of the rats reached "training criterion" defined as total errors (sum of RM and WM errors) per trial as less than one, on a 5-d running average. After reaching this criterion, rats were operated on under sodium pentobarbital anesthesia for electrode implantation. Two electrodes were implanted into the dorsal hippocampus of each side (P3.5, L2.7, 3.5, and $2.5 \mathrm{~mm}$ ventral [V] from the skull surface) (Paxinos and Watson 1986). Using guidance by electrophysiological criteria (Leung et al. 2003), the electrodes were targeted to straddle the CA1 cell layer, with the ventral electrode in stratum radiatum (named L1 or R1) and the dorsal one near the alveus (named L2 or R2). RAM training resumed $1 \mathrm{wk}$ after recovery from surgery, and training was continued for at least $2 \mathrm{wk}$ and until the running average (over five trials) of total $\mathrm{RM}$ and WM errors was less than one per day for at least $5 \mathrm{~d}$. Experimental and control rats were selected randomly from a pair of rats with matched performance before treatment. All matched artifact. pairs of experimental and control rats were run on the RAM on the same days after treatment.

After reaching the last training criteria, the rats were rested for $8 \mathrm{~d}$. On the ninth and tenth day, hippocampal ADs were evoked at hourly intervals in the experimental rats, for 5 ADs each day and a total of 10 ADs in $2 \mathrm{~d}$. An AD was evoked by a high-frequency $100-\mathrm{Hz}$ stimulus train of 1 -sec duration $(0.1 \mathrm{msec}$ duration square pulses). Stimulus pulses were cathodal at a CA1 stratum radiatum electrode, with a screw over the cerebellum serving as anode, and of intensity ranging from 120 to $400 \mu \mathrm{A}$. In each rat, the initial high-frequency stimulus intensity was selected as three times the threshold of the commissural evoked potential. If an $\mathrm{AD}$ was not evoked on the first attempt (which occurred $<5 \%$ of the cases), another stimulus train would be given in $5 \mathrm{~min}$ with the stimulus intensity increased by $50 \mu \mathrm{A}$ until an $\mathrm{AD}$ was evoked. The same train stimulus intensity was kept for all of the stimulations, but the intensity would be increased again for the next stimulation if an evoked AD were $<10 \mathrm{sec}$; the maximal stimulus intensity was fixed at $400 \mu \mathrm{A}$. The control rats were given low-frequency stimulations (LFSs), each consisting of 100 pulses at a similar intensity as the "yoked" experimental rat, but at $0.16 \mathrm{~Hz}$. Hippocampal EEG was recorded from all rats during LFS or AD delivery in order to confirm the presence or absence of an $\mathrm{AD}$ and to measure $\mathrm{AD}$ duration.

Commissural-evoked potentials were recorded at the electrodes in one CA1, following stimulation of the contralateral CA1 (Fig. 1A). The contralateral stimulating electrode was selected as the one that evoked a basal dendritic response, which was negative at or near the excitatory synaptic layer in CA1 (basal dendrites) and positive at the apical dendrites or stratum radiatum (Fig. 1B; Leung and Shen 1995). Average evoked potentials (AEPs; eight sweeps) were recorded at 1.5-2 $\times$ stimulus threshold on $2 \mathrm{~d}$ and $1 \mathrm{~d}$ before stimulation/control treatment, referred to as day -2 and day -1 , respectively. Most, but not all rats in Experiments 1, 2, and 3 showed a commissural basal dendritic response. Other rats with commissural apical dendritic response were also kindled but their AEPs were not included in the analysis.
A

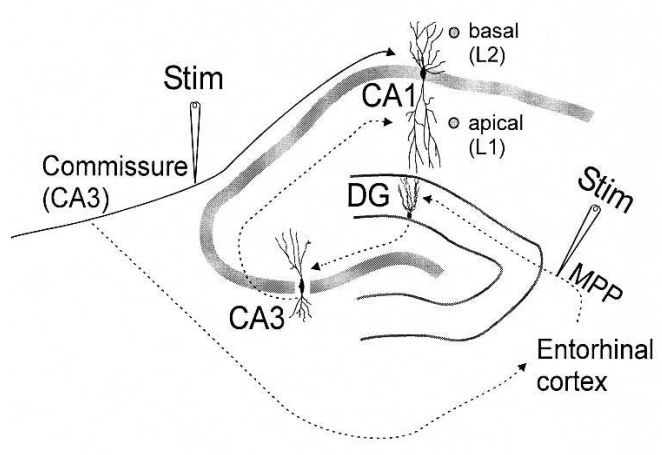

B Commissure (CA3)

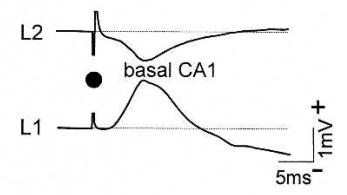

Medial Perforant path (MPP)

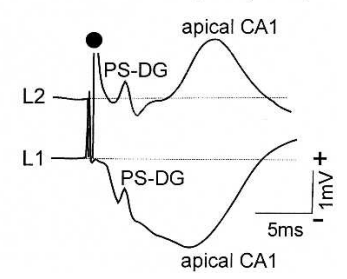

Figure 1. Schematic diagram of the hippocampal circuit studied $(A)$ and the evoked CA1 response (B). (A) Schematic showing stimulation (Stim) of the commissural input (solid trace) from the opposite CA3 (left) to the basal dendrites of CA1. Commissural stimulation also excites the entorhinal cortex (via the dotted trace) that projects as the medial perforant path (MPP). Entorhinal excitation or MPP stimulation excites the dentate gyrus (DG) and then CA3, and then the apical dendrites of CA1. Direct entorhinal excitation of CA3 and CA1 is not illustrated. Filled circles in CA1 indicate depths of electrodes L1 and L2 in CA1, at the apical and basal dendritic layers, respectively. (B) Average evoked potentials (AEPs) recorded at two electrodes in CA1. (Top) Commissural (contralateral CA1) stimulation evoked basal dendritic AEPs in CA1. (Bottom) MPP stimulation evoked an early latency DG population spike (PS-DG) and late polysynaptic apical CA1 excitation. Solid circle indicates time of the stimulus 
Maze trials (2/d) were conducted on days 1 and 4 (wk 1), days 22 and 25 (wk 4), and days 43 and 46 (wk 7) after the last AD. Commissural evoked potentials were recorded within an hour after the maze trial on each day. A sporadic testing schedule was used because previous studies (Leung et al. 1994, 1996) showed that the rats were able to relearn the RAM if testing occurred frequently. At the end of the experiment, rats were deeply anesthetized and perfused through the heart with phosphate-buffered saline, followed by $4 \%$ formaldehyde. Subsequent coronal sections of the brain were sectioned and stained with thionin for confirmation of electrode positions.

\section{Experiment 2. Partial kindling with 5 ADs and spatial performance}

Experiment 2 involved 16 rats designed like Experiment 1, except that the treatment was 5 hippocampal ADs/LFSs in $1 \mathrm{~d}$ instead of 10 ADs/LFSs in $2 \mathrm{~d}$. Rats were implanted with electrodes and then trained to criterion performance on a partially baited RAM. The rats were then rested for $7 \mathrm{~d}$ before stimulation treatment. The experimental rats were given $5 \mathrm{ADs}$ in $1 \mathrm{~d}$, and the control rats were given 5 LFSs in $1 \mathrm{~d}$; ADs or LFSs were given at hourly intervals. Two RAM trials per day were conducted on post-kindling days 1 and 4 (wk 1) and days 22 and 25 (wk 4) after the last AD; wk 7 trials were not conducted. Commissural AEPs were recorded at days -2 and -1 before the ADs/LFSs and on days $1,4,22$, and 25 after the last AD/LFS.

\section{Experiment 3. Perforant path evoked potentials after $10 \mathrm{ADs}$}

In order to investigate whether the CA1 kindling induced synaptic plasticity in the entorhinal cortex-hippocampus circuit (Fig. 1A), an additional group of 16 rats were recorded in CA1 following stimulation of the medial perforant path (MPP). Surgical procedures were similar to Experiment 1, except in addition to the bilateral CA1 electrodes, stimulating electrodes were also placed bilaterally into the angular bundle at P8, L4.4, V3.1 to stimulate MPP. MPP stimulation evoked a population spike in DG. At high-intensity (six times threshold stimulus intensity, averaging $180 \mu \mathrm{A}$ in this group), the latter population spike was evoked at $<3$ msec latency and observed in CA1 following volume conduction (Fig. 1B; Leung et al. 1995). In addition, at 9-12 msec, latency following MPP pulses of $>2 \times$ threshold intensity evoked a negative wave at the CA1 apical dendrites, which reversed to a positive wave at the basal dendrites. The latter CA1 wave was mainly generated by apical dendritic excitation of CA1 following a disynaptic (MPP to CA3 to CA1) or trisynaptic (MPP to DG to CA3 to CA1) pathway; it will be referred to as a polysynaptic MPP excitation of CA1 (Fig. 1).

The rats were trained on both the place and cue RAM (Leung et al. 1996), and assigned to control and experimental groups based on matched RAM performance. The place task was the same as RAM described above. The RAM for the cue task had walls of $16.5 \mathrm{~cm}$ around its perimeter, and different arms of the maze were covered with inserts of different texture (carpet, sandpaper of different roughness, etc.) to enhance the local cues (Leung et al.1996). An opaque curtain completely surrounded the cue maze. Experimental rats were given $10 \mathrm{ADs}$ and control rats given LFSs as in Experiment 1. RAM performance for rats in this Experiment 3 had been reported (Leung et al. 1996) and will not be repeated here. As compared with rats of Experiment 1, the spatial RAM performance of rats in Experiment 3 was relatively poorer before kindling because they were trained simultaneously on both the place and cue RAM tasks. Here, we present the results of MPP-evoked AEPs, which have not been reported before. AEPs were recorded at day -2 and day -1 before ADs/LFSs at $1 \mathrm{~h}$ after the last AD/LFS for the $2 \mathrm{~d}$ of stimulation, and at days $1,5,23$, and 27 after the last AD/LFS.

\section{Experiment 4. HippocampaL LTP in CAl and RAM performance}

The design was similar to Experiment 1, except primed burst stimulations (PBSs) were given instead of high-frequency stimulus trains that evoked ADs. Robust basal dendritic LTP in CA1 was reliably evoked by PBS that did not evoke an AD (Leung et al. 1992). In contrast, apical dendritic synaptic changes were small after PBS of the CA1 apical dendritic pathway (Leung et al. 1992; Leung and Shen 1995). In addition to electrodes in CA1, the rats of this experiment also had stimulating electrodes implanted bilaterally in the MPP.

Twenty rats were trained on the RAM training similar to Experiment 1. Subsequent procedures (detailed later) resulted in exclusion of a few rats, such that the final group used for analysis consisted of seven rats that gave commissural basal dendritic response that was matched in RAM performance with seven control rats. Because of the difficulty of ensuring basal dendritic stimulation and recording in CA1, not all control rats had commissural basal dendritic response. In addition, four rats that gave commissural apical dendritic response were run on the RAM at the same time with the main experimental (basal dendritic response) group and controls.

Rats were trained on the RAM, had surgery for the implantation of electrodes, and then trained again until training criterion was reached. They were rested $7 \mathrm{~d}$ after training criteria before the start of PBSs (experimental group); control rats were handled, but not given any stimulation. All rats in the experimental group $(n=7)$ and three rats in the control group showed a basal dendritic pEPSP in CA1 following contralateral CA1 stimulation. Five of the seven experimental rats and four control rats also had an MPP-evoked response. Twelve PBS trains were given over 2 d, with six trains delivered hourly per day. Each train consisted of eight primed bursts repeated at an interval of 10 sec. One primed burst consisted of a single priming pulse, followed $190 \mathrm{msec}$ later by a burst of 10 pulses at $100 \mathrm{~Hz}$. Stimuli were delivered cathodally to the contralateral CA1 electrode, with the screw over the cerebellum serving as an anode, and the stimulus intensity of the PBS was $3 \times$ threshold of the pEPSP (using $0.1 \mathrm{msec}$ pulse duration). EEG was recorded during the $\mathrm{PB}$ trains to ensure that no $\mathrm{AD}$ was evoked. The rats were tested on week 1 (days 1 and 4) and week 4 (days 22 and 25) after the last PBS train, and at a similar time as the yoked controls. AEPs (average of eight sweeps) in CA1 were recorded on days -1 and -5 before stimulation/control treatment and on days 1, 4, 7, 22, and 25 after treatment. The AEPs were evoked by either contralateral CA1 or MPP stimulation.

\section{Data analysis}

RM and WM errors per trial on the RAM were calculated and averaged for two pretreatment periods as follows: (1) day -7 to day -4 (eight trials, two trials per day) before stimulation/ control treatment, operationally referred to as week -2 , and (2) day -3 to day -1 (six trials, two trials per day) referred to as week -1 before stimulation/control treatment. After stimulation/control treatment, the average RM and WM errors were calculated for (3) first week post-treatment (four trials, two trials each on days 1 and 4 after treatment), (4) fourth week posttreatment (four trials, two trials each on days 22 and 25 after treatment), and (5) seventh week (four trials, two trials each on days 43 and 46 after treatment for Experiment 1 only). For all RAM experiments, a two-factor (group $\times$ time) repeated measures ANOVA was applied to the post-treatment errors after 
Table 1. Average reference memory errors per trial before and after stimulation/control in the different groups

\begin{tabular}{lccccr}
\hline Group & Week $-\mathbf{2}$ & Week -1 & Week 1 & Week 4 & Week 7 \\
\hline Control 10-AD & $0.39 \pm 0.14$ & $0.1 \pm 0.08$ & $1.0 \pm 0.34$ & $0.88 \pm 0.29$ & $1.61 \pm 0.32$ \\
10-AD & $0.3 \pm 0.14$ & $0.25 \pm 0.1$ & $2.28 \pm 0.27^{\mathrm{a}, \mathrm{b}}$ & $1.88 \pm 0.26^{\mathrm{a}, \mathrm{b}}$ & $1.5 \pm 0.31$ \\
Control 5-AD & $0.33 \pm 0.1$ & $0.4 \pm 0.08$ & $0.63 \pm 0.18$ & $0.69 \pm 0.26$ & \\
5-AD & $0.56 \pm 0.15$ & $0.29 \pm 0.09$ & $0.63 \pm 0.08^{\mathrm{b}}$ & $1.13 \pm 0.24^{\mathrm{b}}$ & \\
Control LTP & $0.35 \pm 0.15$ & $0.24 \pm 0.11$ & $0.68 \pm 0.19$ & $1.21 \pm 0.45$ & \\
LTP & $0.23 \pm 0.06$ & $0.19 \pm 0.07$ & $0.69 \pm 0.27$ & $0.88 \pm 0.23$ & \\
\hline
\end{tabular}

Week -2 was the average of the eight trials from -7 to $-4 d$ before stimulation treatment, week -1 was the average of six trials from -3 to $-1 \mathrm{~d}$ before, and weeks 1,4 , and 7 are averages of four trials during the particular week after treatment. Treatment consisted of control group for 10 ADs, 10-AD group, control for 5 ADs, 5-AD group, control for the primed burst stimulation (PBS) or long-term potentiation group and the LTP group given 12 primed-burst train stimulation. All groups had eight rats except LTP, and its control groups had seven rats. Blank entries indicate data were not available.

${ }^{a} P<0.05$, between one experimental group and its control group, post hoc Newman-Keuls test.

b $P<0.05$, Wilcoxon test, between 10-AD and 5-AD groups.

stimulation/control, using only the first and fourth week scores. The scores were either the average RM errors, or the average sum of both RM and WM errors. ANOVA was not presented for the $\mathrm{WM}$ error scores alone, since the WM errors were generally small and often zero. Unless otherwise stated, homogeneity of the variances in ANOVA passed the Levine's test. Post hoc Newman-Keuls test was used only when the ANOVA showed a main or an interaction effect. In addition, nonparametric Wilcoxon statistics were also used for comparing the data before and after stimulation/control treatment. Nonparametric statistics are not dependent on the assumption of data normality or homogeneity of variances. RAM errors before stimulation/control treatment were very low and generally not normally distributed (because many were near zero).

Commissural AEPs were analyzed mainly at the ventral CA1 electrode where the population excitatory postsynaptic potential (pEPSP) was positive. As explained before (Leung and Shen 1999), the pEPSP signal was larger in magnitude at the ventral than dorsal electrode, but both signals showed qualitatively the same change with kindling or LTP. The slope of the pEPSP over a 1-2msec interval was determined, and this interval was fixed for all times for each rat. Stimulation of MPP evoked a population spike generated by the DG, which was analyzed by the valley-to-peak of the volume-conducted signal in CA1 (Fig. 1B). MPP stimulation also evoked a polysynaptic wave in CA1 (Fig. 1B). Most rats showed stable baselines and gradual changes, except immediately after ADs/PBSs, during a 4-wk period of recordings. A few rats with unstable baseline (differing by $>50 \%$ in the two baseline measures) were not included. Occasional rats $(<10 \%)$ in which the stimulus threshold (in $\mu \mathrm{A}$ ) or evoked response changed by more than twofold $(200 \%$ or $50 \%)$ during two successive posttreatment recordings were also excluded from the analysis. A

Table 2. Average working memory errors before and after stimulations in the different groups

\begin{tabular}{lccccc}
\hline Group & Week $-\mathbf{2}$ & Week $-\mathbf{1}$ & Week 1 & Week 4 & Week 7 \\
\hline Control 10-AD & $0.08 \pm 0.05$ & $0.06 \pm 0.06$ & $0.41 \pm 0.14$ & $0.53 \pm 0.23$ & $1.18 \pm 0.35$ \\
10-AD & $0.17 \pm 0.09$ & $0.1 \pm 0.06$ & $1.09 \pm 0.57$ & $0.56 \pm 0.14$ & $0.69 \pm 0.2$ \\
Control 5-AD & $0.25 \pm 0.22$ & $0.06 \pm 0.04$ & $0.31 \pm 0.14$ & $0.59 \pm 0.18$ & \\
5-AD & $0.13 \pm 0.05$ & $0.02 \pm 0.02$ & $0.09 \pm 0.07$ & $0.47 \pm 0.09$ & \\
Control LTP & $0.02 \pm 0.02$ & $0 \pm 0$ & $0.29 \pm 0.14$ & $0.43 \pm 0.17$ & \\
LTP & $0.02 \pm 0.02$ & $0.05 \pm 0.05$ & $0.39 \pm 0.15$ & $0.25 \pm 0.09$ & \\
\hline
\end{tabular}

There was no significant difference among the groups on any day. Rows and columns as explained in the legend of Table 1. All groups had eight rats except LTP group (given primed burst stimulations), and LTP control groups had seven rats. No statistical significance was found among groups at any time, as tested by Wilcoxon test. sudden change of response (other than that immediately after ADs/ PBSs) was considered abnormal, and the change was often accompanied with increasing change in the subsequent days and loss of the head cap.

\section{Results}

\section{Afterdischarges}

The primary AD duration increased from $18.1 \pm 2.2 \mathrm{sec}$ (mean \pm SEM; $n=8)$ for the first $\mathrm{AD}$ to $37.7 \pm 4.7$ sec $(n=8)$ for the fifth $\mathrm{AD}$ in Experiment 2. The $\mathrm{AD}$ duration increased from $36.3 \pm 5.1 \mathrm{sec}$ (first $\mathrm{AD} ; n=8$ ) to $52.5 \pm 13.4 \mathrm{sec}$ (tenth AD) in Experiment 1 and from $30.7 \pm 4.2 \mathrm{sec}$ (first $\mathrm{AD} ; n=8$ ) to $65 \pm 10.9 \mathrm{sec}$ (tenth $\mathrm{AD}$ ) in Experiment 3. No motor convulsion was evoked during kindling. The increase in $\mathrm{AD}$ duration was statistically significant (repeated measure ANOVA, $P<0.05$ ) for Experiments 2 and 3, but not for Experiment 1 . This was partly due to the large fluctuations in $\mathrm{AD}$ duration in three rats in Experiment 1.

\section{Spatial performance after hippocampal ADs}

Experimental and control rats did not differ in their maze performance during acquisition (data not shown) or immediately pre-kindling (Tables 1,2 ). The average $\mathrm{RM}$ errors in periods before treatment were less than one error per trial, and not significantly different $(P>0.05$, Wilcoxon) between control and experimental (10 AD) groups (Fig. 2A; Table 1). RM errors were higher in the kindled than control rats during the first (days 1 and day 4) and fourth week (days 22 and 25) after treatment (Fig. 2), as confirmed by repeated measures ANOVA (main effect $F_{(1,14)}=12$, $P<0.005$; two times, week 1 and week 4 , were used). RM errors on the seventh week were not different between kindled and control rats. Nonparametric Wilcoxon text also yielded a significant difference $(P<0.05)$ between RM errors of control and experimental rats on the first week and fourth week, but not on the seventh week. Combined RM and WM errors gave results similar to RM errors alone. Average WM errors increased somewhat in the experimental rats during the first week postADs (Fig. 2B), but the difference with control rats was not statistically significant $(P>0.05$, Wilcoxon $)$ at this or any other time points.

In Experiment 2 (5-ADs and control groups), there was no difference between experimental and control rats in the initial acquisition of the maze, or in any time point before stimulation treatment (Fig. 2; Tables 1, 2). After kindling/control treatment, average RM errors of both experimental and control groups increased slightly on the first and fourth week, but there was no difference between experimental and control rats. Repeated measure ANOVA of the average RM errors gave $F_{(1,14)}=1.5, P<0.2$ with no time and group $\times$ time interaction. Nonparametric Wilcoxon tests also revealed no significant difference between the 5-AD and its control group at any time before or after stimulation/control. Similarly, analyses of the combined RM and 


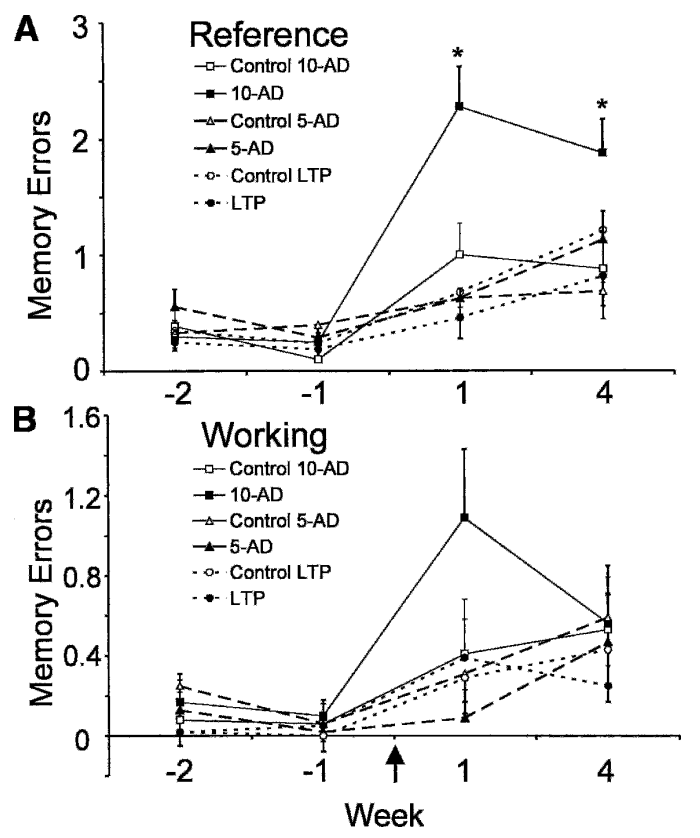

Figure 2. Average reference memory errors $(A)$ and working memory errors $(B)$ on the RAM before and after various stimulation and control procedures. Memory errors are shown for four time periods. Week -2 is operationally defined as the period 4-7 d before stimulation/control treatment. Week -1 is defined as the period 1-3 d before treatment, and week 1 (days 1 and day 4) and week 4 (days 22 and day 25) after treatment. The experimental treatment included 10 ADs, 5 ADs, or LTP (induced by 12 primed burst stimulation trains), each with its control group. Rats were trained on the RAM before stimulation/control treatment. There was no statistical difference between kindled and control group of rats before treatment, and only the change in reference memory error after 10 ADs was significant $\left(\left[{ }^{*}\right] P<0.05\right.$, Wilcoxon), as compared with its controls or to any other group at 1 or $4 \mathrm{wk}$ after treatment.

WM errors or WM errors alone gave no significant difference between 5-AD and its control group at any time.

Synaptic responses were increased by hippocampal ADs Commissural basal dendritic responses in CA1 were found in five of the eight rats given $10 \mathrm{ADs}$ in Experiment 1 . As shown by a representative rat, basal dendritic response was characterized by an initially positive response at the apical dendritic electrode (Fig. 1B; solid trace in Fig. 3), accompanied by a negative response at the basal dendritic electrode (Fig. 1B; dotted trace in Fig. 3). The pEPSPs, as indicated by their slopes (E1 after the first pulse, and E2 after the second pulse), were stable before the start of ADs. Using $100 \%$ as the baseline before kindling, the slope of the pEPSP increased to $159 \%$ after the fifth $\mathrm{AD}$ and $220 \%$ after the tenth $\mathrm{AD}$ in the example shown (Fig. 3). The group data showed a similar trend (Fig. 4). The synaptic enhancement remained high at $1 \mathrm{~d}$ after the ADs, but declined to a smaller value at $4 \mathrm{~d}$ after the ADs (Figs. 3, 4). By $22 \mathrm{~d}$ after the ADs, there was no apparent enhancement in E1 seen in the representative rat or the group average (Fig. 4). Control rats with basal-dendritic responses $(n=5)$ did not show enhancement during or after 10LFSs, and their response at all times remained near 100\% of the baseline. The response of the 10-LFSs control rats was not shown except with the average that included other control rats in Figure 4. Enhancement of E2 (pEPSP slope after the second pulse) was typically smaller after kindling, resulting in a slight decrease in E2/E1 ratio in most rats after kindling.

Rats given 5 ADs showed a similar time course of change of the basal-dendritic pEPSPs as rats after 10 ADs. Synaptic enhance- ment was maximal at $1 \mathrm{~h}$ and $1 \mathrm{~d}$ after the fifth $\mathrm{AD}$ and declined to baseline level by day 22 and day 25 , as shown by a representative rat (Fig. 5) or the group average (Fig. 4).

Recurrent excitation of the hippocampus via the entorhinal cortex (EC) is illustrated by the waveform at $\sim 20 \mathrm{msec}$ latency labeled with an asterisk (*) in Figures 3 and 5. The latter event was interpreted as pEPSP and population spike in the DG, followed by polysynaptic excitation of the apical dendrites of CA1 (Wu et al. 1998). Contralateral CA1 stimulation activated CA3 that subsequently activated EC and then DG, CA3, and CA1, completing a CA3-CA1-EC-DG polysynaptic circuit (Deadwyler et al. 1975; Wu et al. 1998). As shown, the recurrent EC to hippocampus pathway was increased at $1 \mathrm{~h}$ or $1 \mathrm{~d}$ after evoking 5 or 10 ADs.

MPP responses were recorded in seven rats in Experiment 3. One day after 10 ADs evoked by CA1 stimulation, the MPPevoked DG population spike was increased to $148 \pm 16 \%(n=7)$ of the baseline average (Fig. 6), significantly more than $106 \pm 12 \%$ in the controls $(n=5)$. The population spike increase persisted at near 50\% until day 29 after the ADs (Table 3). The difference between 10-AD and control (10-LFS) groups was significant $\left(F_{(1,10)}=5.7, P<0.04\right.$, repeated measures ANOVA, with no significant time effect or group $\times$ time interaction). Similarly, on day 1 after the ADs, the polysynaptic CA1 wave was significantly increased to $137 \pm 11 \%(n=7)$ of its baseline value, but this increase was reduced on day 5 or later after the ADs (Table 3). Commissural CA1 responses recorded in Experiment 3 (data not shown) were similar to those reported for Experiment 1.

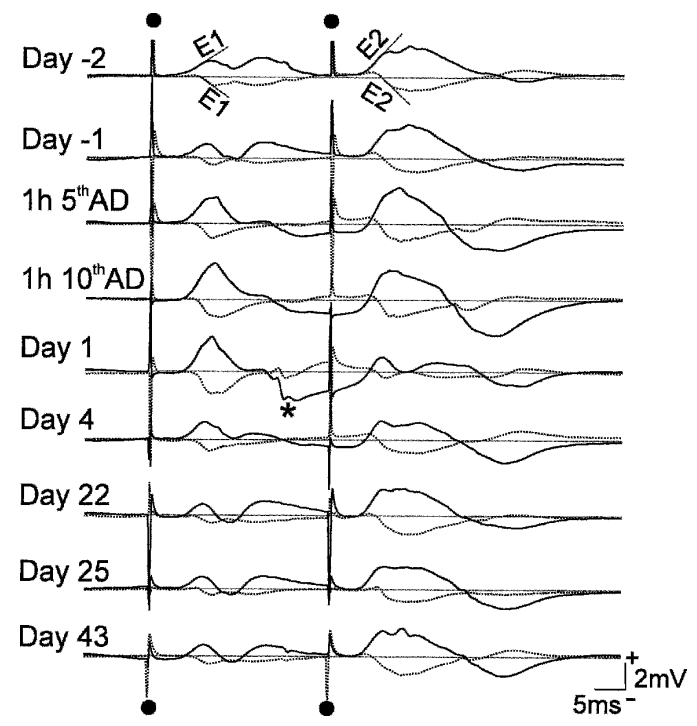

Figure 3. Average evoked potentials (AEPs) in CA1 shows long-lasting enhancement of basal dendritic synaptic transmission in CA1 after 10 hippocampal ADs in a representative rat. Slopes of population excitatory postsynaptic potentials, E1 following the first pulse and E2 following the second pulse, are indicated in the top traces. For the recording on each day, solid trace is AEP recorded at stratum radiatum of CA1 (L1), and dotted trace is AEP recorded near the alveus of CA1 (L2). AEPs were recorded following paired-pulse stimulation (50-msec interpulse interval) of the contralateral (right) CA1 stratum oriens at $120 \mu \mathrm{A}$ intensity. AEPs show an initial positive at electrode $L 1$ and reversed to negative at L2, indicating a basal dendritic response. AEPs were recorded during baseline, $2 \mathrm{~d}$ before (day -2 ) and $1 \mathrm{~d}$ before (day -1$)$ the start of ADs. The ADs were delivered hourly over $2 d$, with 5 ADs given each day. AEPs are shown at $1 \mathrm{~h}$ after the fifth $A D$ and tenth $A D$, and then at days 1, 4, 22, and 25, and 43 after the last AD. The largest enhancement occurred after the fifth and tenth ADs and on day 1 . No enhancement was apparent on day 22 and after. $\left(^{*}\right)$ Volume-conducted dentate gyrus response following recurrent excitation through the entorhinal cortex.

\section{Learning \& Memory \\ www.learnmem.org}




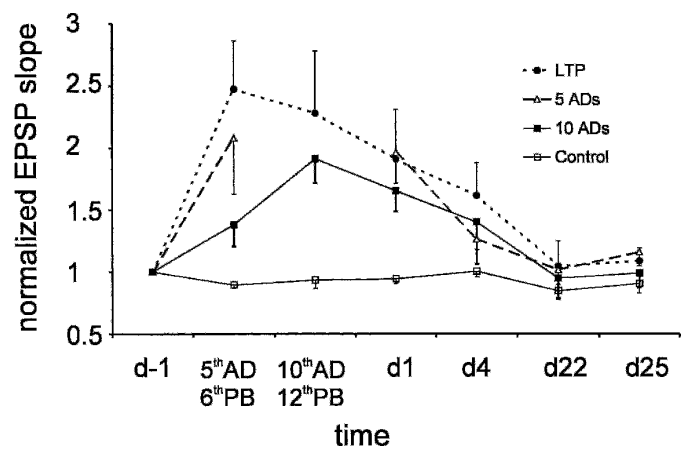

Figure 4. Normalized population EPSP slope shows synaptic enhancement after repeated ADs or PBs that induced LTP. For each rat, the population EPSP slope (E1) during baseline (1 d before stimulations/control treatment or day -1 ) was normalized to one. E1 was measured at $1 \mathrm{~h}$ after the "fifth $A D /$ sixth $P B$ " $\left(5^{\text {th }} A D / 6^{\text {th }} P B\right), 1 \mathrm{~h}$ after "tenth $A D /$ twelfth $\mathrm{PB}^{\prime \prime}\left(10^{\text {th }} \mathrm{AD} / 12^{\text {th }} \mathrm{PB}\right)$, and on day $1(\mathrm{~d} 1)$, day $4(\mathrm{~d} 4)$, day $22(\mathrm{~d} 22)$, and day 25 (d25) after stimulation or control treatment. The 5-AD group had no data at time point "tenth $\mathrm{AD} /$ twelfth $\mathrm{PB}^{\text {". }}$ For simpler presentation, all 12 control rats with basal dendritic E1 were combined into one group, including five rats for 10-AD experiment, three rats for 5-AD experiment, and three rats for the 12-PB (LTP) experiment. Large EPSP slope changes occurred within the first week after stimulation, but not control treatment, irrespective of whether ADs or PBs were given.

\section{Spatial performance after LTP in CAl (Experiment 4)}

Performance on the RAM was matched for experimental (basal dendritic LTP) rats and control rats before PBS/control treatment, and RM and WM errors were not different between experimental and control groups before treatment (Table 1; Fig. 2). Although PBSs were intended not to evoke ADs, one rat showed a total of 4 ADs during the 12 PBSs, and it was removed from further analysis (although its data were not different from the experimental group average). Two other rats showed a single AD during the 2 $\mathrm{d}$ of PBSs; these two rats have been included in the LTP group of seven rats presented below. After PBS/control treatment, RM and WM errors increased slightly, but there was no significant difference between control and LTP rats in any error scores at any time after treatment (Table 1; Fig. 2). Repeated measures ANOVA for the RM errors, or for the combined $\mathrm{RM}+\mathrm{WM}$ errors, gave no significant group effect $\left(F_{(1,12)}<0.84, P>0.3\right)$, time effect, or group $\times$ time interaction. Levine's test of normality was violated $(P<0.05)$ for the RM errors but not the RM + WM errors. Nonparametric Wilcoxon test did not show any difference between control and experimental (basal LTP) groups at any time before or after stimulation/control treatment.

The small group of four rats given PBSs at an apical dendritic synapse in CA1 did not show apical dendritic LTP or any significant difference in spatial performance from control rats at any time before or after PBS/ control treatment.

\section{Synpatic enhancement after primed burst stimulations (LTP)}

Basal-dendritic pEPSP enhancement was shown for the seven rats given PBSs. A twofold increase in pEPSP slope or peak was seen after the sixth or the twelfth PBSs (Fig. 7). The synaptic enhancement of E1 persisted for the first week (up to day 4 in Fig. 7) but was not apparent by days 22 or 25 , either in the example shown (Fig. 7) or the group average (Fig. 4). Responses from control rats $(n=5)$ that were not stimulated remained near $100 \%$ of baseline at all time points of the experiment.

Increase in an evoked population spike after PBSs is shown in the example illustrated (Fig. 7). The increase of the population spike evoked by the first pulse lasted $<4 \mathrm{~d}$ after stimulation, but population spike after the second pulse (i.e., with paired-pulse facilitation) lasted until day 25. Recurrent hippocampal excitation at $\sim 20 \mathrm{msec}$ latency, as indicated by the dentate gyrus population spike (* in Fig. 7), was present after both first and second pulses at $1 \mathrm{~h}$ after PBSs, and only after the second pulse at $1 \mathrm{~d}$ after PBSs.

The DG population spike after MPP stimulation was increased to $150 \pm 14 \%(n=5)$ of the baseline value on $1 \mathrm{~d}$ after the last $\mathrm{PB}$, but returned to baseline ( 100\%) after $1 \mathrm{wk}$ (Table 3$)$. The CA1 polysynaptic wave evoked by MPP stimulation was not increased in the LTP group and it remained at near-baseline level at all times (Table 4). Control rats showed no significant change of the MPP-evoked DG population spike or CA1 wave (Tables 3, 4).

A small group of four rats given PBSs showed a commissural apical dendritic CA1 response. These rats showed no significant change in the apical pEPSPs in CA1 following PBSs. Their RAM performance was also not different from the nonstimulated control rats at any time before and after PBSs/control treatment.

\section{Comparisons among experiments}

The RAM procedures were nearly identical among Experiments 1, 2 , and 4 , and the three control groups (one for each experiment) performed similarly, as indicated by their RM and WM errors at each time point (Fig. 2). The similarity among control groups allows comparisons to be made among experimental groups. RM errors were significantly higher $(P<0.05$, Wilcoxon $)$ in the $10-\mathrm{AD}$ group than in the 5 -AD (or LTP) group during the first and fourth week after kindling. There was no significant difference between the WM errors in the 10-AD and 5-AD (or LTP) group.

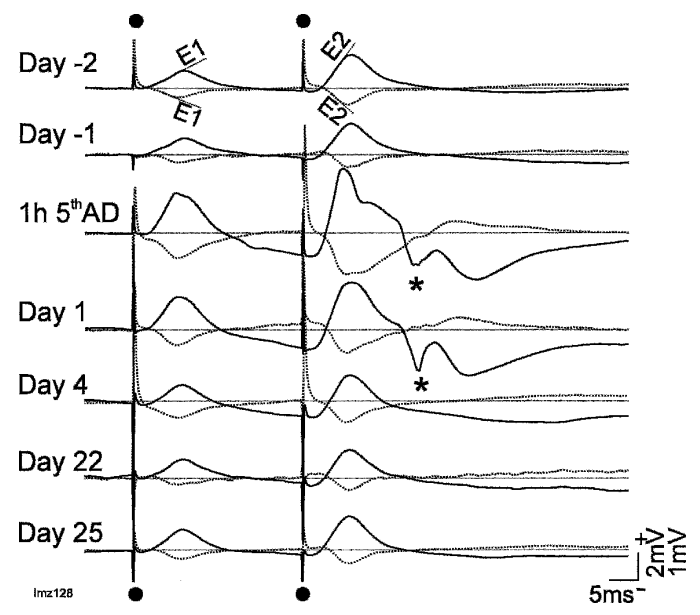

Figure 5. Average evoked potentials (AEPs) in CA1 shows long-lasting enhancement of basal dendritic synaptic transmission in CA1 after 5 hippocampal ADs in one representative rat (Mz128; Experiment 2). Display is similar to Figure 3. Slopes of population excitatory postsynaptic potentials, E1 following the first pulse and E2 following the second pulse, indicated in the top traces. For each day, solid trace is AEP recorded at stratum radiatum of CA1 (L1), and dotted trace is AEP recorded near the alveus of CA1 (L2). AEPs were recorded following paired-pulse stimulation (50-msec interpulse interval) of the contralateral (right) CA1 stratum oriens at $150 \mu \mathrm{A}$ intensity. AEPs were recorded during baseline, $2 \mathrm{~d}$ before $($ day -2$)$ and one day before (day -1$)$ the start of ADs. The ADs were delivered hourly in $1 \mathrm{~d}$. AEPs are shown at $1 \mathrm{~h}$ after the fifth $A D$ and at days 1, 4, 22, and 25 after the last AD. Enhancement of E1 is apparent up to day 4 after ADs, but present in this rat at day $25 .\left(^{*}\right)$ Volume-conducted dentate gyrus response following recurrent excitation through the entorhinal cortex. 


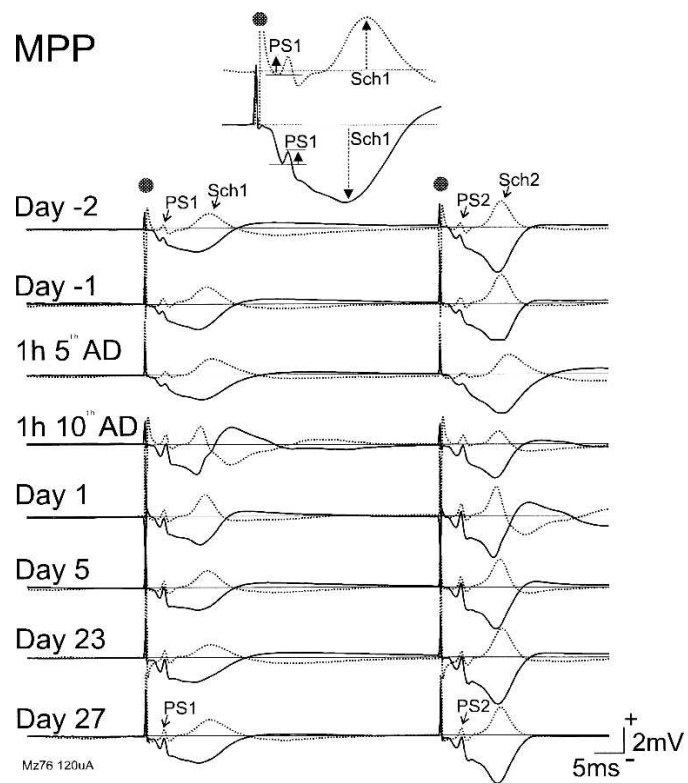

Figure 6. Long-term changes of MPP-evoked responses recorded in CA1 before and after 10 ADs (Mz76 rat; Experiment 3). Expanded recordings at dorsal (top) and ventral (bottom) electrodes in hippocampal CA1 revealed population spike volume conducted from the dentate gyrus (PS1 after the first pulse), and polysynaptic CA1 wave (Sch1) measured as indicated. Display is similar to Figure 3. A persistent (until last record on day 27) enhancement of PS1 and PS2 (population spike following the second pulse) and a less persistent (lasting $<5 \mathrm{~d}$ ) increase in late CA1 wave (Sch1) are shown after 10 ADs.

Increase in the commissural basal-dendritic response showed a time course and peak that were similar among the experimental groups, irrespective of whether 12 PBs (LTP), 5 ADs, or 10 ADs were given (Fig. 4). Further, both AD and LTP groups showed increased recurrent entorhinal-hippocampal excitation at $1 \mathrm{~h}$ and $1 \mathrm{~d}$ after stimulation. Both 10-ADs and LTP groups showed an increase in the population spike in the DG on day 1 and days $4 / 5$, but the increase in DG population spike was significantly larger in the $10-\mathrm{AD}$ than the 12 -PB group ( ${ }^{\mathrm{d}}$ in Table 3 ; Newman-Keuls post hoc test after a significant group $\times$ time interaction). Similarly, the increase of the MPP-evoked polysynaptic CA1 wave (Table 4) was significantly larger after 10-ADs than 12-PBSs (LTP), as revealed by a group effect after repeated measures ANOVA $\left(F_{(1,12)}=5.1, P<0.05\right)$ with no significant time effect or group $\times$ time interaction.

\section{Discussion}

\section{Disruption of spatial performance after seizures}

Using the same training and testing conditions, spatial memory retention deficits lasting about 4 wk were found after 10 but not 5 hippocampal ADs. On the other hand, additional ADs above 10 , such as $15,21,35$ ADs, or $>50$ ADs after full CA1 kindling (Leung et al. 1990, 1994; Leung and Shen 1991) did not appear to increase the severity or duration of the RAM deficits. RAM performance was similar among all control groups in this study, and the control group using repeated LFSs in Experiments 1 and 2 performed similarly as the no-stimulation controls in Experiment 4.

For unknown reasons, the first (and subsequent) AD duration of the 5-AD group was shorter than that of other 10-AD groups (Experiment 1 or 3). The short ADs may contribute to a smaller effect of the ADs on spatial memory disruption, but they do not counter the argument that a certain number of ADs is necessary to disrupt spatial behavior, although an exact minimal number has not been determined.

A disruption of reference memory was shown for week 1 and week 4, but not week 7 after 10 hippocampal ADs (Fig. 2). While relearning the maze was always a factor, it is unlikely that it contributed substantially to the result of week 7 , because lower errors were not found in week 7 as compared with week 4. Further, in another experiment in which only three post-treatment maze trials (instead of eight trials in this study) were run before week 7 , there was also no disruption of week 7 maze performance after kindling (Leung et al. 1994).

This report shows that kindling disrupted reference, but not working memory scores, also reported by Feasey-Truger et al. (1994). The lack of working memory error disruption could be a floor effect, i.e., errors were too small and not sensitive to disruption. Leung et al. (1996) reported a small, but significant disruption of working memory by 10 hippocampal ADs. Memory deficits are found after temporal lobe seizures in humans. Halgren et al. (1991) documented a few cases in which an episode of multiple seizures induced deficit in recent memory of words and faces that persisted for days, similar to the long-lasting deficits in rats reported here.

\section{Basal LTP did not disrupt spatial performance}

A robust basal dendritic LTP in CA1 was induced by repeated PBSs without hippocampal ADs (except for a single AD in two rats), but no spatial memory deficit was detected in rats after basal LTP. As described in the above, various results were found for spatial learning after LTP of the MPP-DG synapse. Basal LTP in CA1 showed 100\% enhancement on day 1 and lasted about $1 \mathrm{wk}$, as

Table 3. Normalized dentate gyrus population spike amplitude evoked by medial perforant path in groups given 10 afterdischarges (ADs) or primed-burst stimulation trains (PBS) and their respective control groups

\begin{tabular}{|c|c|c|c|c|c|c|c|}
\hline Group & $\mathrm{N}$ & $\begin{array}{c}1 \mathrm{~h} \text { post } 1^{\text {st }} \\
\text { day treatment }\end{array}$ & $\begin{array}{l}1 \mathrm{~h} \text { post } 2^{\text {nd }} \\
\text { day treatment }\end{array}$ & Day 1 & Day $4 / 5$ & Day $22 / 23$ & Day $27 / 28$ \\
\hline Control 10-AD & 5 & $1.07 \pm 0.07$ & $1.09 \pm 0.12$ & $1.06 \pm 0.12$ & $0.94 \pm 0.15$ & $1.13 \pm 0.22$ & $1.02 \pm 0.16$ \\
\hline $10-A D^{b}$ & 7 & $0.91 \pm 0.22$ & $1.34 \pm 0.27$ & $1.48 \pm 0.16^{\mathrm{a}}$ & $1.5 \pm 0.14^{\mathrm{a}}$ & $1.43 \pm 0.17^{a}$ & $1.47 \pm 0.1^{a}$ \\
\hline Control LTP & 4 & $1.11 \pm 0.14$ & $0.92 \pm 0.17$ & $1.0 \pm 0.14$ & $1.19 \pm 0.11$ & $0.86 \pm 0.09$ & $0.89 \pm 0.21$ \\
\hline LTP & 5 & $1.2 \pm 0.23$ & $1.25 \pm 0.17$ & $1.5 \pm 0.14^{a, c}$ & $1.3 \pm 0.07^{a}$ & $0.77 \pm 0.19^{d}$ & $0.83 \pm 0.21^{d}$ \\
\hline
\end{tabular}

The average population spike amplitude during the $5 \mathrm{~d}$ immediately before stimulation treatment was used for normalization. The first and second columns are data recorded $1 \mathrm{~h}$ after the last AD or control treatment of the first day and second day, respectively. Rightmost four columns are data after treatment. 10-AD control and experimental rats were recorded on days 5, 23, and 27 after treatment, while long-term potentiation (LTP) rats and their controls were recorded on days 4, 22, and 28 after 12 primed burst stimulation trains or control.

asignificantly different $(P<0.05)$ from baseline before treatment (Wilcoxon).

bSignificantly different $(P<0.05)$ from its own control group (main effect, ANOVA).

'Significantly different $(P<0.05)$ from its own control group, post hoc Newman-Keuls test after significant interaction in ANOVA.

${ }^{\mathrm{d}}$ LTP group significantly different $(P<0.05)$ from 10 -AD group, post hoc Newman-Keuls test after significant interaction in ANOVA.

\section{Learning \& Memory}

www.learnmem.org 


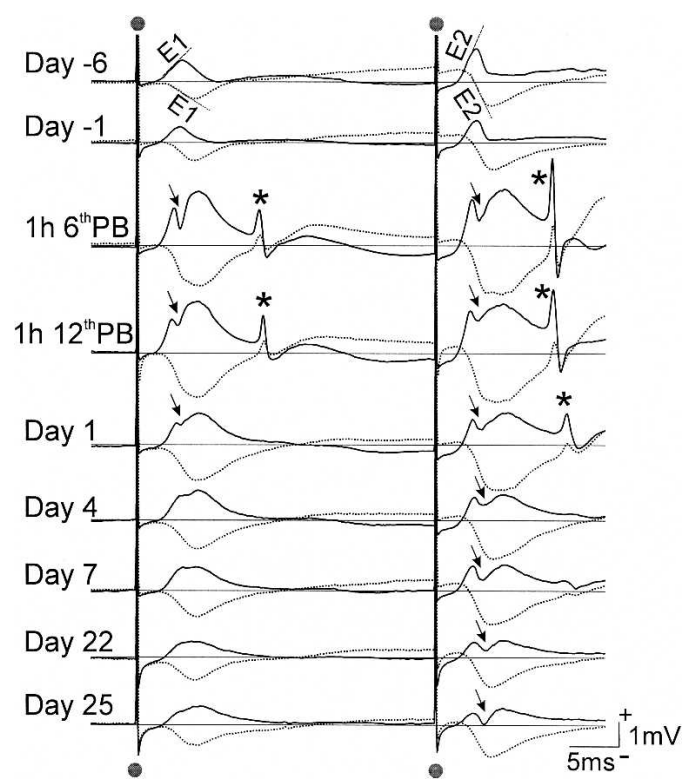

Figure 7. Long-lasting potentiation of responses in CA1 before and after primed-burst stimulation of basal dendritic synapses in a representative rat (Mz85; Experiment 4). Similar layout as Figure 3, with solid trace being AEP recorded at stratum radiatum of CA1 (L1), and dotted trace AEP recorded near the alveus of CA1 (L2). AEPs were recorded following paired-pulse stimulation (30-msec interpulse interval) of the contralateral (right) CA1 stratum oriens at $150 \mu \mathrm{A}$. AEPs were recorded during baseline, $6 \mathrm{~d}$ before (day -6 ) and one day before (day -1 ) the start of primed burst stimulation (PB). The PB trains were delivered hourly over $2 \mathrm{~d}$, with six trains of PB given each day. AEPs are shown at $1 \mathrm{~h}$ after the sixth PB train and twelfth PB train, and then at Days 1, 4, 7, 22, and 25 after the last (twelfth) PB train. The largest potentiation occurred immediately after stimulation, as shown after the sixth and twelfth PB trains, Day 1 and Day 4. Potentiation was quantified by the increase in slopes of the population excitatory postsynaptic potentials, E1 and E2; a population spike was also induced (arrow). Population spike following the second pulse persisted to Day 25, although E1 and E2 returned to near baseline values. $\left(^{*}\right)$ Volume-conducted population spike from the dentate gyrus following recurrent excitation through the entorhinal cortex.

compared with a small enhancement of pEPSPs in the DG (typically <30\%) that lasted several weeks (Barnes et al. 1994). PBSs in CA1 stimulated axons of CA3 cells that project extensively ipsilaterally and contralaterally (Li et al. 1994). However, the degree of saturation and the spatial extent of the LTP in CA1 have not been determined experimentally.

Spatial memory disruption by apical LTP without evoking ADs has not been shown, and repeated PBSs were not successful in inducing apical LTP in this and other studies (Leung et al. 1992; Leung and Shen 1995) (but see Staubli and Lynch 1987). However, repeated hippocampal ADs can increase apical den- dritic synaptic transmission (Leung and Shen 1991), but in addition also induced trans-synaptic alteration of an entorhinal cortex to hippocampus circuit (below).

\section{Neural correlate of kindling induced spatial performance deficit}

This study shows that 10 but not 5 hippocampal ADs resulted in long-lasting spatial memory deficit. This result limits the neural mechanisms that can account for the behavioral deficit. Hippocampal cell death and mossy fiber sprouting are not likely causes of the behavioral deficit, since these required many generalized seizures (Cavazos et al. 1994). We suggested previously (Leung and Shen 1991; Leung et al. 1994, 1996) that retrograde effects on disrupting memory consolidation (Knowlton et al. 1989) is unlikely because the memory in this study should have been consolidated more than $1 \mathrm{wk}$ before kindling. We favor the explanation that 10 ADs cause a change in the hippocampus (e.g., in synaptic transmission) that interferes with recall of a previously stored memory.

The type of altered synaptic transmission that underlies RAM deficits is not clearly known. As reported above, basal dendritic synaptic enhancement alone did not correlate with RAM deficit. Also, basal dendritic synaptic enhancement of similar magnitude was found in rats after 5 ADs, 10 ADs, or basal LTP, but RAM performance was only disrupted after 10 ADs. An increase in recurrent entorhinal-hippocampal excitation also did not correlate with RAM deficit, since it was observed in all experimental groups. However, persistent (1-28 d) changes in the MPP-evoked DG population spike and MPP-evoked polysynaptic wave in CA1 were only observed in rats after $10 \mathrm{ADs}$ but not after LTP (Tables 3, 4). We suggest this distributed effect of hippocampal transmission change may be the basis for the disrupted spatial performance. The increase of the MPP to DG response was a trans-synaptic effect following high-frequency stimulation of CA3 to CA1 afferents. CA1 stimulation would backfire CA3 neurons that projected extensively to both left and right CA1 (Li et al. 1994) and to the EC, subsequently activating the EC-DGCA3-CA1 pathway. Spread of the AD may also increase the spatial extent of the trans-synaptic activation. Repeated ADs also change neural transmission by altering inhibition among other cellular changes (Leung et al. 1994; Lopes da Silva et al. 1995; Mody 1999).

High-frequency tetanization of the MPP was reported to induce trans-synaptic LTP of the DG-CA3 synapse and the CA3-CA1 synapse, but only for $24 \mathrm{~h}$ (Buzsaki 1988). Brun et al. (2001) showed that LTP of the MPP-DG response disrupted retention on the RAM, but MPP to CA1 response was not studied. This report shows that 10, but not 5 hippocampal ADs evoked by CA1 stimulation resulted in long-lasting change in a multisynaptic entorhinal-hippocampal circuit and in disruption of spatial memory retention in rats.

Table 4. Normalized magnitude of polysynaptic apical wave in CA1 evoked by medial perforant path in groups given 10 afterdischarges (ADs) or 12 primed-burst stimulation trains (long-term potentiation or LTP group) and their respective control group

\begin{tabular}{lccccccc}
\hline Group & N & $\begin{array}{c}\text { 1 h post } \mathbf{~}^{\text {st }} \\
\text { day treatment }\end{array}$ & $\begin{array}{c}\text { 1 h post 2 } \\
\text { day treatment }\end{array}$ & Day 1 & Day 4/5 & Day 22/23 & Day 27/28 \\
\hline Control 10-AD & 5 & $1.15 \pm 0.11$ & $1.18 \pm 0.09$ & $1.17 \pm 0.13$ & $1.11 \pm 0.09$ & $1.0 \pm 0.04$ & $1.04 \pm 0.08$ \\
10-AD & 7 & $1.11 \pm 0.14$ & $1.14 \pm 0.14$ & $1.37 \pm 0.11^{\text {a }}$ & $1.18 \pm 0.08$ & $1.19 \pm 0.11$ & $1.06 \pm 0.06$ \\
Control LTP & 4 & $0.84 \pm 0.08$ & $0.88 \pm 0.04$ & $0.79 \pm 0.08$ & $0.84 \pm 0.12$ & $0.91 \pm 0.19$ & $0.83 \pm 0.31$ \\
LTPb & 5 & $1.06 \pm 0.07$ & $0.9 \pm 0.09$ & $0.99 \pm 0.08$ & $0.93 \pm 0.07$ & $0.89 \pm 0.11$ & $0.96 \pm 0.11$ \\
\hline
\end{tabular}

Columns as explained in Table 3.

a Significantly different $(P<0.05)$ from baseline before treatment, Wilcoxon.

bLTP group significantly different $(P<0.05)$ from $10-A D$ group, main effect, repeated measures ANOVA. 


\section{Acknowledgments}

This work was supported by grants from the Natural Sciences and Engineering Research Council of Canada and Canadian Institutes of Health Research grant 64433. We thank Dr. J. Ma for comments on the manuscript and D. Young for technical assistance in Experiment 1.

\section{References}

Bannerman, D.M., Good, M.A., Butcher, S.P., Ramsay, M., and Morris, R.G. 1995. Distinct components of spatial learning revealed by prior training and NMDA receptor blockade. Nature 378: 182-186.

Barnes, C.A., Jung, M.W., McNaughton, B.L., Korol, D.L., Andreasson, K., and Worley, P.F. 1994. LTP saturation and spatial learning disruption: Effects of task variables and saturation levels. J. Neurosci. 14: $5793-5806$.

Brun, V.H., Ytterbo, K., Morris, R.G., Moser, M.B., and Moser, E.I. 2001. Retrograde amnesia for spatial memory induced by NMDA receptor-mediated long-term potentiation. J. Neurosci. 21: 356-362.

Bruton, C.J. 1988. The neuropathology of temporal lobe epilepsy. Oxford Univeristy Press, New York.

Buzsaki, G. 1988. Polysynaptic long-term potentiation: A physiological role of the perforant path-CA3/CA1 pyramidal cell synapse. Brain Res. 45: 192-195.

Cain, D.P., Hargreaves, E.L., Boon, F., and Dennison, Z. 1993. An examination of the relations between hippocampal long term potentiation, kindling, afterdischarge, and place learning in the water maze. Hippocampus 3: 153-164.

Castro, C.A., Silbert, L.H., McNaughton, B.L., and Barnes, C.A. 1989. Recovery of spatial learning deficits after decay of electrically induced synaptic enhancement in the hippocampus. Nature 324: 545-548.

Cavazos, J.E., Das, I., and Sutula, T.P. 1994. Neuronal loss induced in limbic pathways by kindling: Evidence for induction of hippocampal sclerosis by repeated brief seizures. J. Neurosci. 14: 3106-3121.

Deadwyler, S.A., West, J.A., Cotman, C., and Lynch, G. 1975. Physiological studies of the reciprocal connections between the hippocampus and entorhinal cortex. Exp. Neurol. 49: 35-37.

Dragoi, G., Harris, K.D., and Buzsaki, G. 2003. Place representation within hippocampal networks is modified by long-term potentiation. Neuron 39: 843-853.

Feasey-Truger, K.J., Kargl, L., and ten Bruggencate, G. 1993. Differential effects of dentate kindling on working and reference spatial memory in the rat. Neurosci. Lett. 151: 25-28.

Gilbert, T.H., McNamara, R.K., and Corcoran, M.E. 1996. Kindling of hippocampal field CA1 impairs spatial learning and retention in the Morris water maze. Behav. Brain Res. 82: 57-66.

Goddard, G.V., McIntyre, D.C., and Leech, C.K. 1969. A permanent change in brain function resulting from daily electrical stimulation. Exp. Neurol. 25: 295-330.

Halgren, E., Stapleton, J., Domalski, P., Swartz, B.E., Delgado-Excueta, A.V., Walsh, G.O., Mandelkern, M., Blahd, W., and Ropchan, J. 1991. Memory dysfunction in epilepsy patients as a derangement of normal physiology. Adv. Neurol. 55: pp. 385-410.

Hannesson, D.K. and Corcoran, M.E. 2000. The mnemonic effects of kindling. Neurosci. Biobehav. Rev. 24: 725-751.

Hannesson, D.K., Mohapel, P., and Corcoran, M.E. 2001. Dorsal hippocampal kindling selectively impairs spatial learning/short-term memory. Hippocampus 11: 275-286.

Holscher, C., McGlinchey, L., Anwyl, R., and Rowan, M.J. 1997. HFS-induced long-term potentiation and LFS-induced depotentiation in area CA1 of the hippocampus are not good models for learning. Psychopharmacology 130: 174-182.

Knowlton, B.J., Shapiro, M.L., Olton, D.S., and Gamzu, E. 1989. Hippocampal seizures disrupt working memory performance but not reference memory acquisition. Behav. Neurosci. 103: 1144-1147.

Lanke, J., Mansson, L., Bjerkemo, M., and Kjellstrand, P. 1993. Spatial memory and stereotypic behaviour of animals in radial arm mazes. Brain Res. 605: 221-228.

Leung, L.S. and Shen, B. 1991. Hippocampal CA1 evoked response and radial 8-arm maze performance after hippocampal kindling. Brain Res. 555: 353-357.

. 1995. Long-term potentiation of the apical and basal dendritic synapses following local stimulation in CA1 of freely moving rats. $J$. Neurophysiol. 73: 1938-1946.

. 1999. LTP at apical and basal synapses of CA1 in awake rats has different sensitivity to NMDA receptor antagonists. Hippocampus 9: 617-630.
Leung, L.S., Boon, K.A., Kaibara, T., and Innis, N.K. 1990. Radial maze performance following hippocampal kindling. Behav. Brain Res. 40: $119-129$.

Leung, L.S., Shen, B., and Kaibara, T. 1992. Long-term potentiation induced by patterned stimulation of the commissural pathway to hippocampal CA1 region in freely moving rats. Neuroscience 48: $63-74$.

Leung, L.S., Zhao, D., and Shen, B. 1994. Long-lasting effects of partial hippocampal kindling on hippocampal physiology and function. Hippocampus 4: 696-704.

Leung, L.S., Roth, L., and Canning, K. 1995. Entorhinal inputs to the hippocampal CA1 and dentate gyrus in the rat: A current-source-density study. J. Neurophysiol. 73: 2392-2403.

Leung, L.S., Brzozowski, D., and Shen, B. 1996. Partial hippocampal kindling affects retention but not acquisition, place but not cue task on the radial arm maze. Behav. Neurosci. 110: 1017-1024.

Leung, L.S., Ma, J., and McLachlan, R.S. 2000. Behaviors induced or disrupted by complex partial seizures. Neurosci. BioBehav. Rev. 24: 763-775.

Leung, L.S., Shen, B., Ma, J., and Rajakumar, N. 2003. Cholinergic activity enhances hippocampal long-term potentiation during walking in rats. J. Neurosci. 23: 9297-9304.

Li, X.G., Somogyi, P., Ylinen, A., and Buzsaki, G. 1994. The Hippocampal Ca3 network-An in vivo intracellular labeling study. J. Comp. Neurol. 339: 181-208.

Lopes da Silva, F.H., Gorter, J.A., and Wadman, W.J. 1986. Kindling of the hippocampal induces spatial memory deficits in the rat. Neurosci. Lett. 63: 115-120.

Lopes da Silva, F.H., Kamphuis, W., Titulaer, M., Vreugdenhil, M., and Wadman, W.J. 1995. An experimental model of progressive epilepsy: The development of kindling of the hippocampus of the rat. Ital. J. Neurol. Sci. 16: 45-57.

McNamara, R.K., Kirkby, R.D., dePape, G.E., Skelton, R.W., and Corcoran, M.E. 1993. Differential effects of kindling and kindled seizures on place learning in the Morris water maze. Hippocampus 3: $149-152$.

Mody, I. 1999. Synaptic plasticity in kindling. Adv. Neurol. 79: 631-643.

Moser, E.I., Krobert, K.A., Moser, M.B., and Morris, R.G. 1998. Impaired spatial learning after saturation of long-term potentiation. Science 281: 2038-2042.

Muller, R.U., Stead, M., and Pach, J. 1996. The hippocampus as a cognitive graph. J. Gen. Physiol 107: 663-694.

Nosten-Bertrand, M., Errington, M.L., Murphy, K.P., Tokugawa, Y. Barboni, E., Kozlova, D., Michalovich, D., Morris, R.G., Silver, J., Stewart, C.L., et al. 1996. Normal spatial learning despite regional inhibition of LTP in mice lacking Thy-1. Nature 379: 826-829.

Olton, D.S., Becker, J.T., and Handelman, G.E. 1979. Hippocampus, space, and memory. Behav. Brain Sci. 2: 313-322.

Paxinos, G. and Watson, C. 1986. The rat brain in stereotaxic coordinates, 2nd ed. Academic Press. San Diego, CA.

Robinson, G.B. 1992. Maintained saturation of hippocampal long-term potentiation does not disrupt acquisition of the 8-arm radial maze. Hippocampus 2: 389-395.

Robinson, G.B., McNeil, H.A., and Reed, R.D. 1993. Comparison of short- and long-lasting effects of perforant path kindling on radial maze learning. Behav. Neurosci. 6: 1-8.

Saucier, D. and Cain, D.P. 1995. Spatial learning without NMDA receptor-dependent long-term potentiation. Nature 378: 186-189.

Staubli, U. and Lynch, G. 1987. Stable hippocampal long-term potentiation elicited by " $\theta$ " pattern stimulation. Brain Res. 435: $227-234$

Sutherland, R.J., Leung, L.S., Weisend, M.P., Schlife, J., and McDonald, R.J. 1997. An evaluation of the effect of partial hippocampal kindling on place navigation by rats in the Morris water task. Psychobiology 25: 126-132.

Sutula, T., Lauersdorf, S., Lynch, M., Jurgella, C., and Woodard, A. 1995. Deficits in radial arm maze performance in kindled rats: Evidence for long-lasting memory dysfunction induced by repeated brief seizures. J. Neurosci. 15: 8295-8301.

Thompson, P.J. 1991. Memory function in patients with epilepsy. Adv. Neurol. 55: 369-384.

Tsien, J.Z., Huerta, P.T., and Tonegawa, S. 1996. The essential role of hippocampal CA1 NMDA receptor-dependent synaptic plasticity in spatial memory. Cell 87: 1327-1338.

$\mathrm{Wu}$, K., Canning, K.J., and Leung, L.S. 1998. Functional interconnections between CA3 and the dentate gyrus revealed by current source density analysis. Hippocampus 8: 217-230.

Received August 25, 2005; accepted in revised form October 17, 2005.

\section{$26 \quad$ Learning \& Memory}




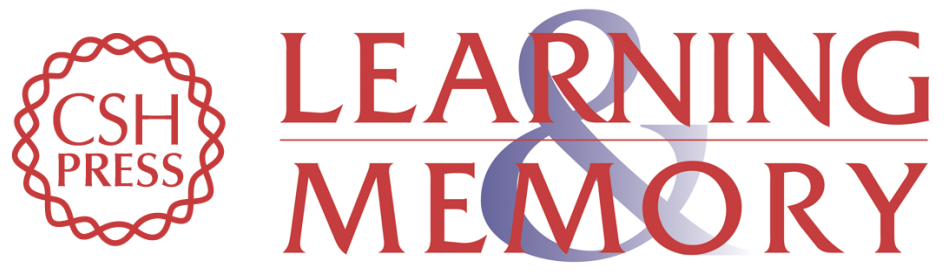

\section{Hippocampal CA1 kindling but not long-term potentiation disrupts spatial memory performance}

L. Stan Leung and Bixia Shen

Learn. Mem. 2006, 13:

Access the most recent version at doi:10.1101/lm.66106

References This article cites 44 articles, 7 of which can be accessed free at:

http://learnmem.cshlp.org/content/13/1/18.full.htmI\#ref-list-1

License

Email Alerting Receive free email alerts when new articles cite this article - sign up in the box at the Service top right corner of the article or click here. 